\title{
Melanoma stem cells: the past, present and future
}

\begin{abstract}
Metastatic melanoma is a cancer that is notoriously difficult to treat due to its ability to form resistance to various treatment regimens. Cancer stem cells represent a population of tumor cells capable of self-renewal and differentiation, capable of maintaining the tumor, and capable of resisting treatment. Melanoma stem cells have also been identified with increasing amounts of information being elucidated about these cells over the past decade. Therapeutic targeting of stem cells will be a key component in the fight against malignant melanoma and other cancers in the future.
\end{abstract}

\author{
Volume 4 Issue 4 - 2018
}

\author{
Peter Chow,' Sarah Moore,' Gaurav \\ Kaushik ${ }^{2}$ \\ 'The University of Kansas, School of Medicine, USA \\ ${ }^{2}$ Department of Surgery, The University of Kansas Medical \\ Center, USA
}

\begin{abstract}
Correspondence: Gaurav Kaushik (PHD), The University of Kansas Medical Center, Department of Surgery, 390I Rainbow Boulevard, Kansas City, KS, 66160, USA,
\end{abstract}

Email gauravkaushik2I@gmail.com

Received: August 02, 20I8 | Published: August 24, 2018

\section{Introduction}

Normal adult stem cells are characterized by their ability to selfrenew, as well as their ability to differentiate into various mature cell types. Cancer stem cells were first recognized by Bonnet et al, who showed a sub-portion of acute myelogenous leukemia (AML) stem cells could be identified and separated from AML cells in patients. This subset of AML cells were the only cells capable of transferring AML from the human patients to studied mice. ${ }^{1}$ The hypothesis of the existence of cancer stem cells gained more attention around 2001 when Reya et al pushed the notion that cancer stems cells are composed of a subset fraction of tumor cells that have the ability to maintain the tumor through self-renewal, conferring drug resistance, and inducing tumor relapse. ${ }^{2}$ These cancer stem cells have similar physiologic properties to normal adult stem cells, like self-renewal and differentiation. Normal stem cells had been shown to be resistant to cytotoxic agents compared to mature cell types, which is explained by anti-apoptotic mechanisms, ${ }^{3}$ quiescence, ${ }^{4}$ and high expression levels of ATP-binding cassette (ABC) transporters. ${ }^{5}$ Thus, the existence of cancer stem cells explains the reason why many treatments for metastatic tumors ultimately fail. Current treatments like chemotherapy and radiation can shrink, but not cure metastatic tumors. Frequently, these treatments do indeed target the bulk of tumor cells in the human body, but often, the drug therapy is unable to kill the cancer stem cells (due to inherited and/or acquired resistance), and thus the tumor can easily grow back. In recent years, cancer stem cells have been identified and isolated by characteristics of normal stem cells, like using tissue specific CD markers (The cluster of differentiation) and $\mathrm{ABC}$ transporter proteins. ${ }^{6}$ Research about targeted therapy in regard to these cancer stem cells has come in full swing over the past decade.

\section{Melanoma stem cells}

Melanoma is a heterogenous skin cancer arising from melanocytes and is notorious for forming resistance to chemotherapy and targeted therapy in its advanced stages. One theory to explain this heterogeneity is the cancer stem cell hypothesis. As cancer stem cells have been defined by their ability to self-renew and differentiate, melanoma stem cells (MSCs)-also called malignant melanoma initiating cells-also followed this definition. Fang et al in 2005 were the first to report the presence of melanoma stem cells. These MSCs were enriched in the CD20 surface marker, normally a marker of mature B lymphocytes. These CD20+ melanoma cells were capable of self-renewal and differentiation. ${ }^{7}$ In 2008, Schatton et al demonstrated ABCB5, an $\mathrm{ABC}$ transporter protein known to confer drug resistance, as the first functional putative biomarker for melanoma stem cells. ${ }^{8}$ Many other markers were discovered for MSCs after this, including CD133, CD271 and ALDH. ${ }^{9}{ }^{10}$ However, more research and exploration is required to establish exclusive markers for MSCs.

MSCs are unique in their immune evasion techniques. MSCs that express $\mathrm{ABCB} 5$ are associated with decreased expression of melanoma antigen recognized by T cells-1 (MART-1), which is associated with melanocyte differentiation and $\mathrm{T}$ cell recognition, in order to evade host T-cell recognition. ${ }^{11}$ MSCs also evade the immune system with altered expression of major histocompatibility complex and inhibition of interleukin-2 (IL-2), two components critical in host immunity. ${ }^{11}$

Considering the knowledge gained about MSCs and their existence, there are multiple future outlets to explore in regard to the treatment of melanoma and stem cells. First, melanoma stem cell specific markers can be detectable targets using monoclonal antibodies. Since MSCs express CD20, rituximab, a CD20 antibody therapy, has been attempted in clinical trials to treat melanoma, producing regression in chemotherapy-refractory melanoma. ${ }^{12}$ Similarly, a monoclonal antibody against $\mathrm{ABCB}^{+} \mathrm{MSCs}$ in mouse models showed tumor inhibitory effects. ${ }^{8}$

Given that MSCs block IL-2 as a mechanism of immune evasion, future therapy can focus on regulators of the immune response. For example, cytotoxic T-lymphocyte antigen-4 (CTLA-4) is a receptor associated with the ligand B7-2 upregulated by ABCB5 MSCs, and combined, they negatively regulate host T-cell activation. ${ }^{13}$ Indeed, anti-CTLA4 monoclonal antibodies, ipilimumab and tremelimumab have shown promise in clinical trials ${ }^{11,14}$ in patients with metastatic melanoma. Tuccito et al.$^{15}$ were able to demonstrate another effective means to modifying the immunologic tumor microenvironment. They demonstrated that interleukin-6 (an inflammatory cytokine) released from differentiated melanoma cells reduced MSC self-renewal and instead induced differentiation. Interleukin-10 (an anti-inflammatory 
cytokine) release on the other hand, promoted MSC self-renewal. By silencing interluekin- 10 by blocking the IL-10 receptor. Tuccito et al. ${ }^{15}$ were able to sensitize MSCs to interleukin-6 and thus induce MSC differentiation. Through these cytokine manipulations, they were able to decrease MSC self-renewal capacity, and instead differentiate stem cells to a more easily targeted phenotype. ${ }^{15}$

$\mathrm{Du}$ et al have recently shown how to use stem cells as an ally (rather than an enemy) in treatment of metastatic melanoma to the brain. In the mouse model, they successfully demonstrated the utility of mesenchymal stem cells to carry oncolytic herpes simplex virus through intracarotid administration to metastatic brain lesions and deliver the oncolytic viruses. This is important in the future treatment of metastatic melanoma because of the paucity of options and high mortality associated with metastatic melanoma to the brain. ${ }^{16}$

\section{Conclusion}

Malignant melanoma is a difficult to treat cancer due to its heterogenous subpopulations of cells. The awareness of the existence of melanoma stem cells will be critical in the future to combat melanoma. Future therapy can be targeted at melanoma stem cell biomarkers, microenvironment, or even using stem cells as a treatment option themselves. Nevertheless, much more research in melanoma and stem cells will be necessary to fully add clarity to treatment plans.

\section{Acknowledgements}

None.

\section{Conflicts of interest}

Authors declare no conflicts of interest.

\section{References}

1. Bonnet D, Dick JE. Human acute myeloid leukemia is organized as a hierarchy that originates from a primitive hematopoietic cell. Nat Med. 1997;3(7):730-737.

2. Reya T, Morrison SJ, Clarke MF, et al. Stem cells, cancer, and cancer stem cells. Nature. 20011;414(6859):105-111.

3. Peters R, Leyvraz S, Perey L. Apoptotic regulation in primitive hematopoietic precursors. Blood. 1998;92(6):2041-2052.
4. Harrison DE, Lerner CP. Most primitive hematopoietic stem cells are stimulated to cycle rapidly after treatment with 5-fluorouracil. Blood. 1991;78(5):1237-1240.

5. Zhou S, Schuetz JD, Bunting KD, et al. The ABC transporter Bcrp1/ABCG2 is expressed in a wide variety of stem cells and is a molecular determinant of the side-population phenotype. Nat Med. 2001;7(9):1028-1034.

6. Garvalov BK, Acker T. Cancer stem cells: a new framework for the design of tumor therapies. J Mol Med (Berl). 2011;89(2):95-107.

7. Fang D, Nguyen TK, Leishear K, et al. A tumorigenic subpopulation with stem cell properties in melanomas. Cancer Res. 2005;65(20):9328-9337.

8. Schatton T, Murphy GF, Frank NY, et al. Identification of cells initiating human melanomas. Nature. 2008;451(7176):345-349.

9. Civenni G, Walter A, Kobert N, et al. Human CD271-positive melanoma stem cells associated with metastasis establish tumor heterogeneity and long-term growth. Cancer Res. 2011;71(8):3098-3109.

10. Luo Y, Dallaglio K, Chen Y, et al. ALDH1A isozymes are markers of human melanoma stem cells and potential therapeutic targets. Stem Cells. 2012;30(10):2100-2113.

11. Murphy GF, Wilson BJ, Girouard SD, et al. Stem cells and targeted approaches to melanoma cure. Mol Aspects Med. 2014;39:33-49.

12. Schlaak M, Schmidt P, Bangard C, et al. Regression of metastatic melanoma in a patient by antibody targeting of cancer stem cells. Oncotarget. 2012;3(1):22-30.

13. Schatton T, Schütte U, Frank NY, et al. Modulation of T-cell activation by malignant melanoma initiating cells. Cancer Res. 2010;70(2):697-708.

14. Wolchok JD, Neyns B, Linette G, Negrier S, et al. Ipilimumab monotherapy in patients with pretreated advanced melanoma: a randomised, double-blind, multicentre, phase 2, dose-ranging study. Lancet Oncol. 2010;11(2):155-164.

15. Tuccitto A, Tazzari M, Beretta V, et al. Immunomodulatory Factors Control the Fate of Melanoma Tumor Initiating Cells. Stem Cells. 2016;34(10):2449-2460.

16. Du W, Seah I, Bougazzoul O, et al. Stem cell-released oncolytic herpes simplex virus has therapeutic efficacy in brain metastatic melanomas. Proc Natl Acad Sci. 2017;114(30):E6157-E6165. 